\title{
ON THE COMPOSITION OF FINITE ROTATIONS IN $\mathbb{E}^{4}$
}

\author{
ALEX GOLDVARD AND LAVI KARP
}

Communicated by Gregory L. Naber

\begin{abstract}
We achieve compositions rules for the geometric parameters of the composed rotations, which is in a certain sense analogous to the well known Rodrigues formula. We also obtain a necessary and sufficient condition for a composition of two simple rotations in $\mathbb{E}^{4}$ to be a simple rotation.
\end{abstract}

MSC: 14L35, 15B10, 51F25, 51N30

Keywords: Clifford translation, Gibbs vector, orthogonal planes, quaternionic representation, simple and double rotations

\section{Introduction}

In this note we are studying compositions of finite rotations in the Euclidean space $\mathbb{E}^{4}$, the four dimensional real vector space with the standard scalar product.

There are several significant differences between rotations in $\mathbb{E}^{4}$ and in the three dimensional space. The group of rotations in $\mathbb{E}^{3}$ essentially comprises one type, that is, a rotation about an axis, while in $\mathbb{E}^{4}$ there are three types of rotations: i) A simple rotation, it leaves a plane (two dimensional subspace of $\mathbb{E}^{4}$ ) point-wise fixed and induces a two dimensional rotation in the orthogonal plane; ii) A Clifford translation (an isoclinic rotation), here each vector in $\mathbb{E}^{4}$ turns through the same angle; iii) A double rotation, here $\mathbb{E}^{4}$ is decomposed into two orthogonal planes and points in the first plane rotate through an angle $\alpha$, while points in the second plane rotate through an angle, $\beta \neq \alpha$.

Here we derive two properties of the compositions of rotations in $\mathbb{E}^{4}$. The first one gives analytical and geometrical characterizations of the subgroup of simple rotations. The second result deals with compositions formulas for double rotations, that is, we obtained formulas that enable the calculations of the orthogonal planes and angles of the composed rotation in terms of the corresponding characteristics of each one of the rotations in $\mathbb{E}^{4}$.

Our main tool is the quaternionic representation

$$
x \mapsto a x b
$$


of rotations in $\mathbb{E}^{4}$. Cayley obtained formula (1) in 1855 by means of laborious calculations [2]. Many years later, in 1945, Coxeter [4] gave a very elegant proof of the above formula by employing compositions of reflections to represent rotations. He also derived the geometric characterization of the rotation (1), that is, he computed the orthogonal planes and angles via the quaternions $a$ and $b$. But there are several cases in which Coxter's formulas are not valid. We shall fill the gap in this note and complete the geometric characterization in those cases.

The outline of the paper is as follows. In the following section we shall give a brief account of Coxeter's approach and treat the above mentioned special cases. Section 3 deals with the composition of simple rotations. In Section 4 we calculate planes and angles of a composed rotation and exhibit an example. We end with Section 5 where we discuss the results.

\section{Representation of Rotations and Reflections by Quaternions}

We first fix the notations. A quaternion $x$ is expressed as $x=x_{0}+x_{1} \mathrm{i}+x_{2} \mathrm{j}+x_{3} \mathrm{k} \equiv$ $S x+V x$, where $S x=x_{0}$ is called the real part, $V x=x_{1} \mathrm{i}+x_{2} \mathrm{j}+x_{3} \mathrm{k}$ is the vector part, and the coefficients $x_{0}, x_{1}, x_{2}$ and $x_{4}$ are real numbers. The quaternions multiplication is defined by the table

$$
\mathrm{i}^{2}=\mathrm{j}^{2}=\mathrm{k}^{2}=-1, \quad \mathrm{ij}=-\mathrm{ji}=\mathrm{k}, \quad \mathrm{jk}=-\mathrm{kj}=\mathrm{i}, \quad \mathrm{ki}=-\mathrm{ik}=\mathrm{j} .
$$

We denote by $\mathbb{H}$ the set of all quaternions. Since a quaternion is completely defined by its four real components, it determines a point (vector) in the four dimensional real space $\mathbb{R}^{4}$. Similarly, $V x=x_{1} \mathrm{i}+x_{2} \mathrm{j}+x_{3} \mathrm{k}$ can be identified with a point (vector) in three dimensional space $\mathbb{R}^{3}$. The multiplication formula

$$
x y=S x S y-V x \cdot V y+S x V y+S y V x+V x \times V y
$$

is a consequence of the distributive property of the quanternionic algebra. Here $V x \cdot V y$ and $V x \times V y$ are the scalar and cross products of vectors in $\mathbb{E}^{3}$.

Let $x$ be a quaternion, then $\bar{x}=S x-V x$ is the conjugate quaternion, and $N x=$ $\bar{x} x=x \bar{x}=x_{0}^{2}+x_{1}^{2}+x_{2}^{2}+x_{3}^{2}$ is the square of the norm. When $N x=1$, then it is called a unit quaternion. Any unit quaternion $a$ can be written in a polar form $a=\cos \alpha+\sin \alpha p$, where $p=p_{1} \mathrm{i}+p_{2} \mathrm{j}+p_{3} \mathrm{k}$ and satisfies $p^{2}=-1$. The vector $p$ is called a pure unit quaternion. A subspace which is spanned by two quaternions $a$ and $b$ is denoted by $\operatorname{Sp}\{a, b\}=\left\{s_{1} a+s_{2} b ; s_{1}, s_{2} \in \mathbb{R}\right\}$.

The advantage of quaternionic representation relies on two simple properties of quaternions. The function $f(x)=a x$ is linear and $N(a x)=N a N x$. Therefore, if $a$ is a unit quaternion, then the multiplication $a x$ represents an orthogonal transformation in $\mathbb{E}^{4}$. Moreover, for any unit quaternion $x,(a x) \cdot x=S(a x \bar{x})=S(a)$, 
hence the cosine of the angle between $a x$ and $x$ is equal to $S(a)$ for any $x \in \mathbb{E}^{4}$. Thus any vector in $\mathbb{E}^{4}$ rotates with angle $\alpha$, where $\cos \alpha=S(a)$. Such type of rotations does not exist in $\mathbb{E}^{3}$, and it is called a left Clifford translation. It is evident that the set of all left Clifford translations forms a subgroup of the rotation group in $\mathbb{E}^{4}$. Similarly, one may consider a right Clifford transformation $x \mapsto x b$, where $b$ is a unit quaternion. Now, the composition of left and right Clifford translations results in the formula (1). As we already mentioned, Coxeter proved that this formula comprises all rotations in $\mathbb{E}^{4}$ by using reflections with respect to hyperplanes. For readers' convenient we recall his description of reflections and rotations.

\section{Reflections}

Notice that any two quaternions $y$ and $x$ are orthogonal in $\mathbb{E}^{4}$ if and only if $x \bar{y}+$ $y \bar{x}=0$. Hence if $N y=1$, then the transformation

$$
x \mapsto-y \bar{x} y
$$

is a reflection with respect to a hyperplane having $y$ as its normal (see $[4, \S 5])$. In what follows we denote this reflection by $R_{y}$.

\section{Simple Rotations}

A rotation in the plane through the angle $\alpha$ and all the points in the orthogonal plane remain fixed. Its representation is given by

$$
x \mapsto a x b, \quad N a=N b=1, \quad S a=S b=\cos \frac{\alpha}{2} .
$$

It is shown in [4] that there are two unit quaternions $y$ and $z$ such that $a=z \bar{y}, b=$ $\bar{y} z$, and the simple rotation (3) is the composition of the reflections $R_{y}(x)=-y \bar{x} y$ and $R_{z}(x)=-z \bar{x} z$, that is

$$
x \mapsto a x b=(z \bar{y}) x(\bar{y} z) .
$$

Obviously, $x \mapsto(z \bar{y}) x(\bar{y} z)=x$, whenever $x$ is orthogonal both to $y$ and $z$. Hence, the point-wise invariant plane of the simple rotation (4) is the intersection of the hyperplanes

$$
\Pi_{1}:=\left\{x \in \mathbb{H} ; \sum_{\mu=0}^{3} y_{\mu} x_{\mu}=0\right\}, \quad \Pi_{2}:=\left\{x \in \mathbb{H} ; \sum_{\mu=0}^{3} z_{\mu} x_{\mu}=0\right\} .
$$




\section{Clifford Translations}

Each vector in $\mathbb{E}^{4}$ turns through the same angle. Let $a$ be a unit quaternion, then $x \mapsto a x$ is a left Clifford translation and $x \mapsto x a$ is a right Clifford translation. The angle $\alpha$ of both types satisfies the equation $\cos \alpha=S a$.

Writing $a$ in a polar form $a=\cos \alpha+\sin \alpha p$, and consider the left translation $x \mapsto a x$, then

$$
x \mapsto x \cos \alpha+p x \sin \alpha, \quad p x \mapsto p x \cos \alpha-x \sin \alpha .
$$

Hence the plane $\operatorname{Sp}\{x, p x\}$ is an invariant subspace of this transformation, and consequently Clifford translations have infinite number of invariant two dimensional subspaces. Similarly, for the right Clifford translation $x \mapsto x b, b=\cos \beta+q \sin \beta$, the planes $\operatorname{Sp}\{x, x q\}$ are invariant subspaces. These subspaces are completely defined by the pure quaternions $p$ and $q$ of the Clifford translations. In addition, we observe the similarity between (5) and the formula for rotations in $\mathbb{E}^{2}$.

\section{Double Rotations}

Two orthogonal planes rotate simultaneously with two different angles. Let $p$ and $q$ be two pure quaternions such that $p^{2}=q^{2}=-1$, then a double rotation has the representation

$$
x \mapsto(\cos \alpha+p \sin \alpha) x(\cos \beta+q \sin \beta)=: f(x) .
$$

The rotation angles are $\alpha \pm \beta$. In case that $\cos \alpha=\cos \beta$, then the formula (6) represents a simple rotation.

Note that if $\cos \alpha, \cos \beta \neq 0$, then we can set $\tilde{p}=\tan \alpha p, \tilde{q}=\tan \beta q$ and rewrite formula (6) as

$$
f(x)=\cos \alpha(1+\tilde{p}) x \cos \beta(1+\tilde{q}) .
$$

We will use this representation in Section 4. The quaternions $\tilde{p}$ and $\tilde{q}$ are called Gibbs vectors. We shall call (7) Gibbs representation of the rotation.

\section{The Orthogonal Planes of the Double and Simple Rotations}

Coxeter proved that the orthogonal planes of the rotation (6) are

$$
\Pi_{1}=\operatorname{Sp}\{p-q, 1+p q\} \quad \text { and } \quad \Pi_{2}=\operatorname{Sp}\{p+q, 1-p q\} .
$$

Points in the plane $\Pi_{1}$ rotate through the angle $\alpha+\beta$ and in the plane $\Pi_{2}$ through the angle $\alpha-\beta$, see $[4, \S 9]$. It follows that if $\beta=\alpha$, then (6) is a simple rotation with the points-wise invariant plane $\Pi_{2}$, while $\Pi_{1}$ is the fixed-plane when $\beta=-\alpha$. 
If one of the quaternions $p \mp q, 1 \pm q$ is zero, then Coxeter's characterization of the invariant planes is not longer valid and we have to treat these cases separately. First of all note that the equalities $p= \pm q$ and $p q=\mp 1$ are equivalent and, in fact, there are only two special cases: $q= \pm p$. It is readily follows from formula (6) that in these cases

$$
f(1) \in \operatorname{Sp}\{1, p\} \quad \text { and } \quad f(p) \in \operatorname{Sp}\{1, p\} .
$$

Therefore $\Lambda_{1}=\operatorname{Sp}\{1, p\}$ is one of the rotating planes. Since the second one $\Lambda_{2}$, is the orthogonal complement of $\Lambda_{1}$, we conclude that

$$
\Lambda_{2}=\{x \in \mathbb{H} ; p \cdot x=S x=0\} .
$$

The pointwise invariant planes of simple rotations when $q= \pm p$ can be found by the following considerations. If $q=p$, then by $f(1) \neq 1$, and therefore $\Lambda_{2}$ is the fixed plane. While if $q=-p$, then $f(1)=1$, and hence $\Lambda_{1}$ is the fixed plane.

\section{Composition of Simple Rotations in $\mathbb{E}^{4}$}

Let $f: x \mapsto a x b$ and $g: x \mapsto c x d$ be two simple rotations and consider the composition $g \circ f: x \mapsto(a c) x(b d)$. We ask under which conditions $g \circ f$ will be again a simple rotation.

It follows from (3) that the rotation $g \circ f$ is simple if and only if $S(c a)=S(b d)$. According to the multiplication formula (2), this condition is equivalent to

$$
S c S a-V c \cdot V a=S b S d-V b \cdot V c .
$$

Since both $f$ and $g$ are simple rotations, $S(a)=S(b)$ and $S(c)=S(d)$, hence the above condition results in

$$
V c \cdot V a-V b \cdot V d=0 .
$$

We now recall that each rotation is decomposed into two reflections by formula (4). Hence, there exists four unit quaternions $y, z, u, w$ such that

$$
a=z \bar{y}, \quad b=\bar{y} z, \quad c=w \bar{u}, \quad d=\bar{u} w .
$$

Hence

$$
\begin{aligned}
V c \cdot V a & =V(w \bar{u}) \cdot V(z \bar{y}) \\
& =\left(-w_{0} V u+u_{0} V w+V u \times V w\right) \cdot\left(-z_{0} V y+y_{0} V z+V y \times V z\right)
\end{aligned}
$$


and

$$
\begin{aligned}
V b \cdot V d & =V(\bar{y} z) \cdot V(\bar{u} w) \\
& =\left(y_{0} V z-z_{0} V y-V y \times V z\right) \cdot\left(u_{0} V w-w_{0} V u-V u \times V w\right) .
\end{aligned}
$$

Thus (8) becomes

$$
\begin{aligned}
V c \cdot V a-V b \cdot V d= & -2\left(y_{0} V z \cdot(V u \times V w)-z_{0} V y \cdot(V u \times V w)\right. \\
& \left.+u_{0} V w \cdot(V y \times V z)-w_{0} V u \cdot(V y \times V z)\right) \\
= & -2 \operatorname{det}[y, z, u, w]=0
\end{aligned}
$$

where $[y, z, u, w]$ denote the matrix with the column vectors $y, z, u$ and $w$. These vectors are the normals of the hyperplanes of the reflections $R_{y}, R_{z}, R_{u}$ and $R_{w}$ that decompose the rotations. Thus we have obtained the following theorem.

Theorem 1. Let $f: x \mapsto a x b$ and $g: x \mapsto c x d$ be two simple rotations such that $f$ is the composition of the reflections $R_{y}$ with $R_{z}$, and $g$ is the composition of the reflections $R_{u}$ with $R_{w}$. Then the composition $g \circ f$ is a simple rotation if and only if the four normals of the reflections are linearly dependent.

The point-wise invariant planes of the rotations $f$ and $g$ are

$$
\Pi_{f}:=\left\{x \in \mathbb{H} ; \sum_{\mu=0}^{3} y_{\mu} x_{\mu}=\sum_{\mu=0}^{3} z_{\mu} x_{\mu}=0\right\}
$$

and

$$
\Pi_{g}:=\left\{x \in \mathbb{H} ; \sum_{\mu=0}^{3} u_{\mu} x_{\mu}=\sum_{\mu=0}^{3} w_{\mu} x_{\mu}=0\right\} .
$$

Obviously, if $\Pi_{f} \cap \Pi_{g} \neq\{0\}$, then the composition $g \circ f$ is simple. However, the converse implication is not trivial. It follows from the above Theorem, that if the composition is simple, then the four normals are linearly dependent, which implies that the intersection $\Pi_{f} \cap \Pi_{g}$ contains a non-zero vector. So a consequence of the Theorem 1 is

Corollary 2. Let $f: x \mapsto a x b$ and $g: x \mapsto c x d$ be two simple rotations, and $\Pi_{f}$ and $\Pi_{g}$ be the point-wise invariant planes of the rotations $f$ and $g$ respectively. Then the composition $h=g \circ f$ is a simple rotation if and only if $\Pi_{f} \cap \Pi_{g} \neq\{0\}$.

Remark 3. When the paper was written the authors were not aware that in 1890 Cole proved Corollary 2. Cole essentially worked out Cayley's parameters of the subgroup and this method demands laborious computations. He also obtained formulas for the orthogonal planes and the angle of the composed rotation, but they are complicated and lengthy, and inapplicable even in some very simple examples. 


\section{The Angles and Planes of the Composed Rotation}

In this section we consider the following problem: Does there exist formulas that link the orthogonal planes and angles of two rotations to those of composed rotation. We start with double and simple rotations? So suppose that

$$
\begin{aligned}
& f(x)=a x b=\cos \alpha_{1}\left(1+\tilde{p}_{1}\right) x\left(1+\tilde{q}_{1}\right) \cos \beta_{1} \\
& g(x)=c x d=\cos \alpha_{2}\left(1+\tilde{p}_{2}\right) x\left(1+\tilde{q}_{2}\right) \cos \beta_{2}
\end{aligned}
$$

are two double rotations such that

$$
\cos \alpha_{1}, \cos \beta_{1} \neq 0 \quad \text { and } \cos \alpha_{2}, \cos \beta_{2} \neq 0 .
$$

Recall that right hand side of (10) and (11) are Gibbs representation of the rotations $f$ and $g$. Consider the composition $f$ followed by $g$

$$
h(x):=(g \circ f)(x)=(c a) x(b d)=\cos \alpha(1+\tilde{p}) x(1+\tilde{q}) \cos \beta .
$$

Then in accordance to the multiplication formula (2)

$$
\cos \alpha=S(c a)=\cos \alpha_{1} \cos \alpha_{2}\left(1-\tilde{p}_{1} \cdot \tilde{p}_{2}\right)
$$

and

$$
p \sin \alpha=V(c a)=\cos \alpha_{1} \cos \alpha_{2}\left(\tilde{p}_{2}+\tilde{p}_{1}+\tilde{p}_{2} \times \tilde{p}_{1}\right) .
$$

Assuming

$$
\cos \alpha \neq 0
$$

we obtain that

$$
\tan \alpha p=\tilde{p}=\frac{\tilde{p}_{2}+\tilde{p}_{1}+\tilde{p}_{2} \times \tilde{p}_{1}}{1-\tilde{p}_{2} \cdot \tilde{p}_{1}}
$$

In a similar manner

$$
\begin{gathered}
\cos \beta=S(b d)=\cos \beta_{1} \cos \beta_{2}\left(1-\tilde{q}_{1} \cdot \tilde{q}_{2}\right) \\
\sin \beta q=V(b d)=\cos \beta_{1} \cos \beta_{2}\left(\tilde{q}_{1}+\tilde{q}_{2}+\tilde{q}_{1} \times \tilde{q}_{2}\right)
\end{gathered}
$$

and if

$$
\cos \beta \neq 0
$$

then

$$
q \tan \beta=\tilde{q}=\frac{\tilde{q}_{1}+\tilde{q}_{2}+\tilde{q}_{1} \times \tilde{q}_{2}}{1-\tilde{q}_{1} \cdot \tilde{q}_{2}} .
$$

Thus in a view of those computations, we have obtained the following theorem. 
Theorem 4. If the conditions (12), (14) and (17) hold, then the composition of the rotations (10) with (11), $h:=g \circ f$, is a rotation through the angles $\alpha \pm \beta$ about the planes spanned by quaternions $\tilde{p} \tan \alpha \pm \tilde{q} \tan \beta$ and $\tan \alpha \tan \beta \mp \tilde{p} \tilde{q}$, where $\alpha$, $\beta, \tilde{p}$ and $\tilde{q}$ are given by (13) and (16), (15) and (18) respectively.

Note that the angels of the composed rotation are independent of the order of the composition, that is, $g \circ f$ and $f \circ g$ result in the same angles. While the crossproduct in (15) and (18) depend upon the order.

Remark 5. The expressions (15) and (18) resemble the known Rodrigues formula for the composition of rotations in $\mathbb{E}^{3}$ (see e.g. [7, p. 58, 71]). That is, if $f$ is a rotation through an angle $\alpha_{1}$ about an axis $c_{1}$, and $g$ is a rotation through an angle $\alpha_{2}$ about an axis $c_{2}$, then their composition $g \circ f$ ( $f$ followed by $g$ ) is a rotation through an angle $\alpha$ about an axis $c$, where

$$
\tilde{c}=\frac{\tilde{c}_{2}+\tilde{c}_{1}+\tilde{c}_{2} \times \tilde{c}_{1}}{1-\tilde{c}_{2} \cdot \tilde{c}_{1}}
$$

$\tilde{c}:=\tan \frac{\alpha}{2} c, \tilde{c_{1}}:=\tan \frac{\alpha_{1}}{2} c_{1}$ and $\tilde{c_{2}}:=\tan \frac{\alpha_{2}}{2} c_{2}$. The similarity of the formulas (15), (18) and (19) is not accidental. A rotation in $\mathbb{E}^{3}$ about an axis $c$ through an angle $\alpha$ has the quanternionic representation $x \mapsto$ axa , where $a=\cos \frac{\alpha}{2}+$ $c \sin \frac{\alpha}{2}$. Therefore, the formula (19) is obtained in the same way as we do it for the composition of two double rotations in $\mathbb{E}^{4}$. The vector $\tilde{c}$ is called Gibbs vector after J. W. Gibbs who invented it in 1881 in order to achieve formula (19). In fact this formula was obtained by Olinde Rodrigues in 1840, see [6] for the historical survey.

Remark 6. Note that for a rotation in $\mathbb{E}^{3}, \tilde{c}=\tan \frac{\alpha}{2}$ c. Hence the discontinuity occurs when $\alpha= \pm \pi$, which means that the composed rotation is a reflection. For rotations in $\mathbb{E}^{4}$ there are many possibilities for discontinuity. For example, in the case of a composition of simple rotations, where $\cos \alpha=0$ in (13), then it results in a reflection in one plane, and the identity in the orthogonal plan.

Remark 7. To the authors' knowledge Fedorov was the first one who discovered compositions rules of four dimensional rotations in 1958. He obtained compositions' rules for two Lorentz transformations in the Minkowski space, and for rotations in the Euclidean space $\mathbb{E}^{3}$ he stated that analogous formulas to (15) and (18) hold. He applied these results to relativity and quantum physics. A comprehensive treatment of these matters is given in Fedorov's book [5]. Fedorov analyzed the group of Lorentz transformations and showed that it can be parametrized by two arbitrary three dimensional vectors. Writing explicitly the matrix of Lorentz 
transformations via those vectors, he derived the compositions formulas [5, §24]. So Fedorov basically used matrix approach as opposed to the quaternions algebra. He did not consider the geometrical characterization (orthogonal planes and angles). Recently Fedorov's ideas on using Gibbs parametrization of a rotation (it is also called a vector parametrization) became quite widespread, see for example [1] and [8] for further discussions.

We turn now to the composition of two Clifford translations of the same type. So suppose $f: x \mapsto a x$ and $g: x \mapsto b x$ are two left Clifford translations, and consider the composition $f$ followed by $g: h=g \circ f: x \mapsto c x$, where $c=b a$. Writing the quaternions by means of Gibbs vectors, that is, $a=\left(1+\tilde{p}_{1}\right) \cos \alpha_{1}$, $b=\left(1+\tilde{p}_{2}\right) \cos \alpha_{2}$ and $c=(1+\tilde{p}) \cos \alpha$, then by the quanternionic algebra we get that

$$
\tilde{p}=\frac{\tilde{p}_{1}+\tilde{p}_{2}+\tilde{p}_{2} \times \tilde{p}_{1}}{1-\tilde{p}_{1} \cdot \tilde{p}_{2}}
$$

and

$$
\cos \alpha=\left(1-\tilde{p}_{1} \cdot \tilde{p}_{2}\right) \cos \alpha_{1} \cos \alpha_{2} .
$$

Thus in view of formulas (13), (15), (16), (18) and (19) and the two last ones, we may say that in a certain sense all type of rotations obey the same composition rule.

\subsection{Example}

The following example illustrates the consequences of Corollary 2 and Theorem 4 . Let

$$
f: x \mapsto \frac{1+\mathrm{i}}{\sqrt{2}} x \frac{1+\mathrm{j}}{\sqrt{2}} \quad \text { and } \quad g: x \mapsto \frac{1+\mathrm{j}}{\sqrt{2}} x \frac{1+\mathrm{k}}{\sqrt{2}}
$$

be two simple rotations. Then $\Pi_{f}=\operatorname{Sp}\{\mathrm{i}-\mathrm{j}, 1+\mathrm{k}\}$ is the fixed-points plane of the rotation $f$ and $\Pi_{g}=\operatorname{Sp}\{\mathrm{j}-\mathrm{k}, 1+\mathrm{i}\}$ of $g$. We see that $\Pi_{f} \cap \Pi_{g} \neq\{0\}$, hence by Corollary 2 the composition $g \circ f$ should be a simple rotation. Simple calculations show that

$$
h=g \circ f: x \mapsto \frac{1+\mathrm{i}+\mathrm{j}-\mathrm{k}}{2} x \frac{1+\mathrm{i}+\mathrm{j}+\mathrm{k}}{2}=: a x b .
$$

Since $S a=S b$, the composition $g \circ f$ is simple. We shall now verify formulas (13), (15) and (18). In order to it we represent these rotations by the Gibbs vectors as in (7). So let $\tilde{p}_{f}, \tilde{q}_{f}, \tilde{p}_{g}, \tilde{q}_{g}, \tilde{p}_{h}, \tilde{q}_{h}$ the Gibbs vectors that corresponding those rotations, and let $\alpha_{f}, \alpha_{g}, \alpha_{h}$ be angles of Gibbs vectors $(\tilde{p}=p \tan \alpha$, where $p$ is a pure unit quaternion). Then 


$$
\begin{aligned}
& f: x \mapsto\left(\cos \frac{\pi}{4}(1+\mathrm{i})\right) x\left((1+\mathrm{j}) \cos \frac{\pi}{4}\right) \\
& g: x \mapsto\left(\cos \frac{\pi}{4}(1+\mathrm{j})\right) x\left((1+\mathrm{k}) \cos \frac{\pi}{4}\right)
\end{aligned}
$$

and

$$
h: x \mapsto\left(\cos \frac{\pi}{3}(1+\mathrm{i}+\mathrm{j}-\mathrm{k})\right) x\left((1+\mathrm{i}+\mathrm{j}+\mathrm{k}) \cos \frac{\pi}{3}\right) .
$$

By (13)

$$
\cos \alpha_{h}=(1-(\mathrm{i} \cdot \mathrm{j})) \cos \alpha_{f} \cos \alpha_{g}=\left(\cos \frac{\pi}{4}\right)^{2}=\frac{1}{2} .
$$

Thus $\alpha_{h}=\frac{\pi}{3}$, which obviously coincides with (20). Now, in accordance with formulas (15) and (18)

$$
\tilde{p}_{h}=\frac{\mathrm{j}+\mathrm{i}+\mathrm{j} \times \mathrm{i}}{1-\mathrm{j} \cdot \mathrm{i}}=\mathrm{i}+\mathrm{j}-\mathrm{k} \quad \text { and } \quad \tilde{q}_{h}=\frac{\mathrm{j}+\mathrm{k}+\mathrm{j} \times k}{1-\mathrm{j} \cdot \mathrm{k}}=\mathrm{i}+\mathrm{j}+\mathrm{k} \text {. }
$$

\section{Conclusions}

There are various methods to represent rotations in $\mathbb{E}^{4}$. In this article we are using the quaternionic algebra approach. It enables us to obtain the characterization of a nontrivial subgroup of the simple rotations. Another advantage is the quaternionic formula (6) for double rotations that describes in a very intrinsic way the geometric parameters. Relying upon that formula we had achieved the compositions rules by means of very simple calculations.

\section{References}

[1] Brezov M., Mladenova C. and Mladenov I., Vector Decompositions of Rotations, J. Geom. Symmetry Phys. 28 (2012) 67-103.

[2] Cayley A., Recherches ultérieurs sur les déterminants gauches, J. Reine Angew. Math. 50 (1855) 299-313.

[3] Cole F., On Rotations in Space of Four Dimensions, Am. J. Math. 12 (1890) 191-210.

[4] Coxeter H. S. M., Quaternions and Reflections, Amer. Math. Monthly 53 (1946) 136-146.

[5] Fedorov F., Lorentz Group (in Russian), Nauka, Moscow 1979. 
[6] Gray J., Olinde Rodrigues' Paper of 1840 on Transformation Groups, Archive for History of Exact Sciences 21 (1980) 375-385.

[7] Lounesto P., Clifford Algebras and Spinors, $2^{\text {nd }}$ Edn, Cambridge University Press, Cambridge 2001.

[8] Muller A., Group Theoretical Approaches to Vector Parameterization of Rotations, J. Geom. Symmetry Phys. 19 (2010) 43-72.

Received 13 June 2015

Alex Goldvard

Department of Mathematics

ORT Braude College

P.O. Box 78

2161002 Karmiel, ISRAEL

E-mail address: karp@braude.ac.il

Lavi Karp

Department of Mathematics

ORT Braude College

P.O. Box 78

2161002 Karmiel, ISRAEL

E-mail address: goldvard@braude.ac.il 